\title{
MAYO CLINIC \\ Technical Tips for Percutaneous Ablation of Challenging Abdominal Tumors
}

\%)

M.R. Moynagh, FFRRCSI; D. Hill, M.D.; A.N. Kurup, M.D.; J. Schmitz, M.D. ; P. Eiken, M.D.; G. Schmit, M.D.; T. Atwell, M.D. Mayo Clinic, Rochester, MN, USA

Full eposter avaliable at EE-086

Background

Percutaneous tumor ablation gaining acceptance
as a minimally invasive treatment for the management of liver, kidney, prostate and

As the popularity of these procedures
increases, so have the recognized

increases, so have the recognized
challenges of treatment and complications.

Over recent years, several techniques have

and outcomes.

Tumor locations that were previously considered
high-risk, in close proximity to vital structures,

are now more accessible and amenable to

Displacement - Fluid
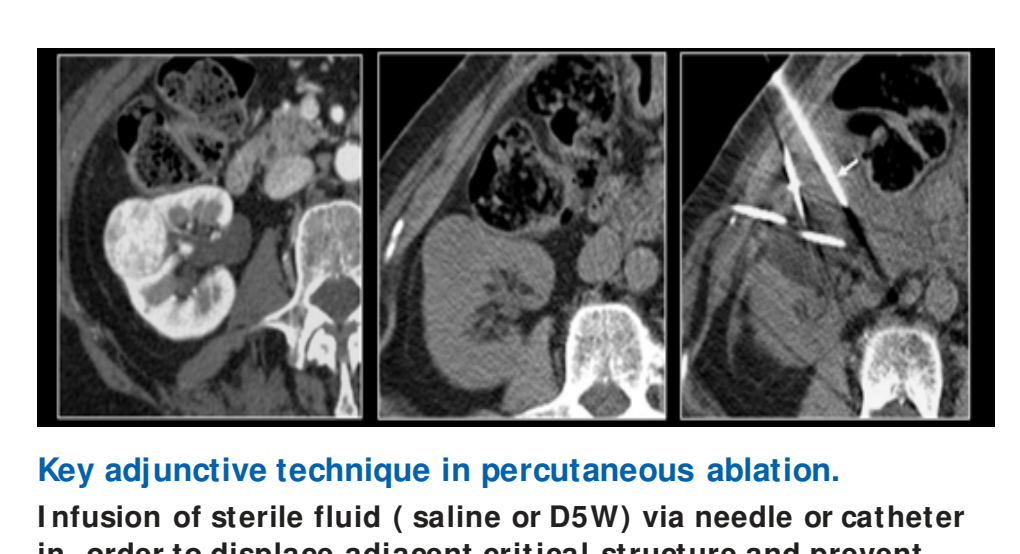
Infusion of sterili flluid ( saline or D5W) via needle or cathete
in urder to displace adjacent critical structure and prevent
ablation of non target structures. In the case shown, sterile ablation of non target structureses In the case shown, sterile
saline has been institlled to tosplacec colon away from target
renal tumor allowing safe growth of ice ball during freeze

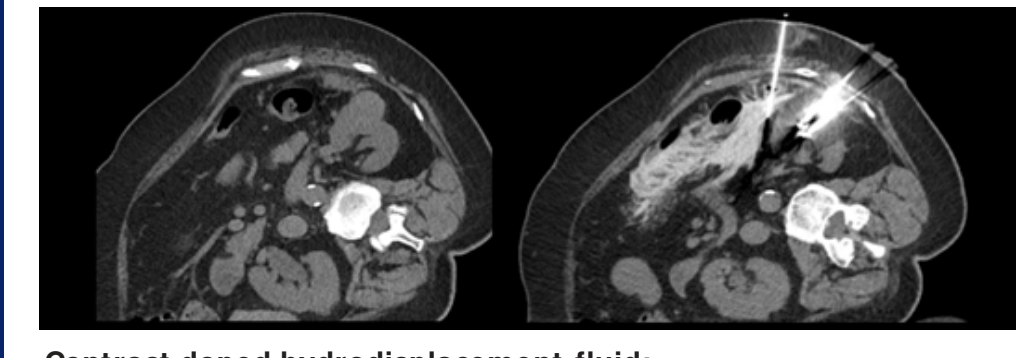

Contrast doped hydrodisplacement fluid:

Non-ionic or ionic contrast media can be added to the sterile
infusion fluid prior to injection with suggested optimal ratio $1: 50 / 2 \%$. This can provided added visualization of adjacent
$1: 50$

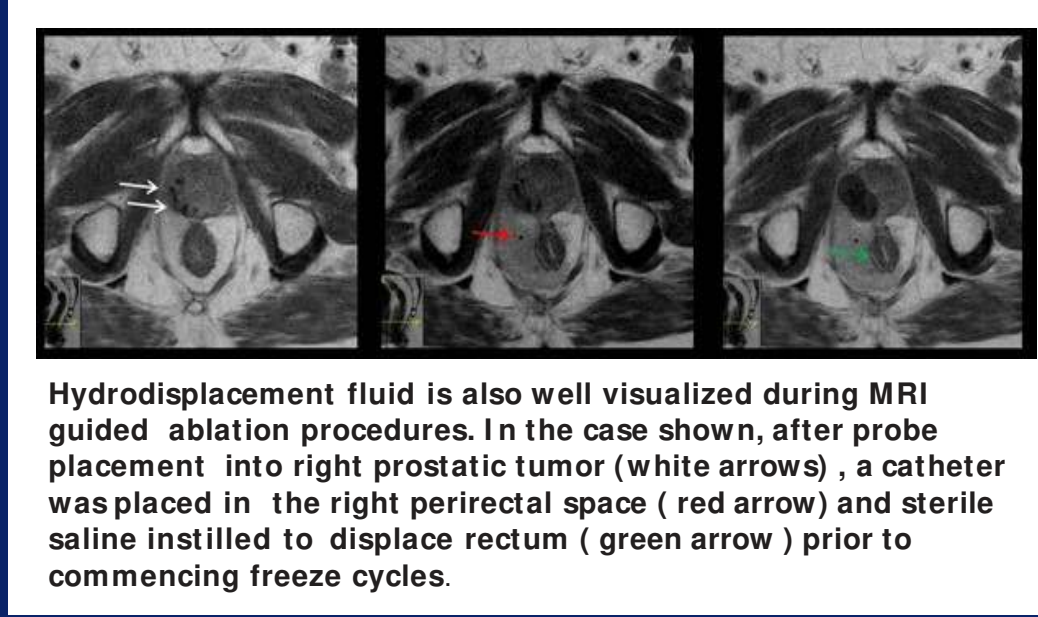

Pre ablation tumor embolization
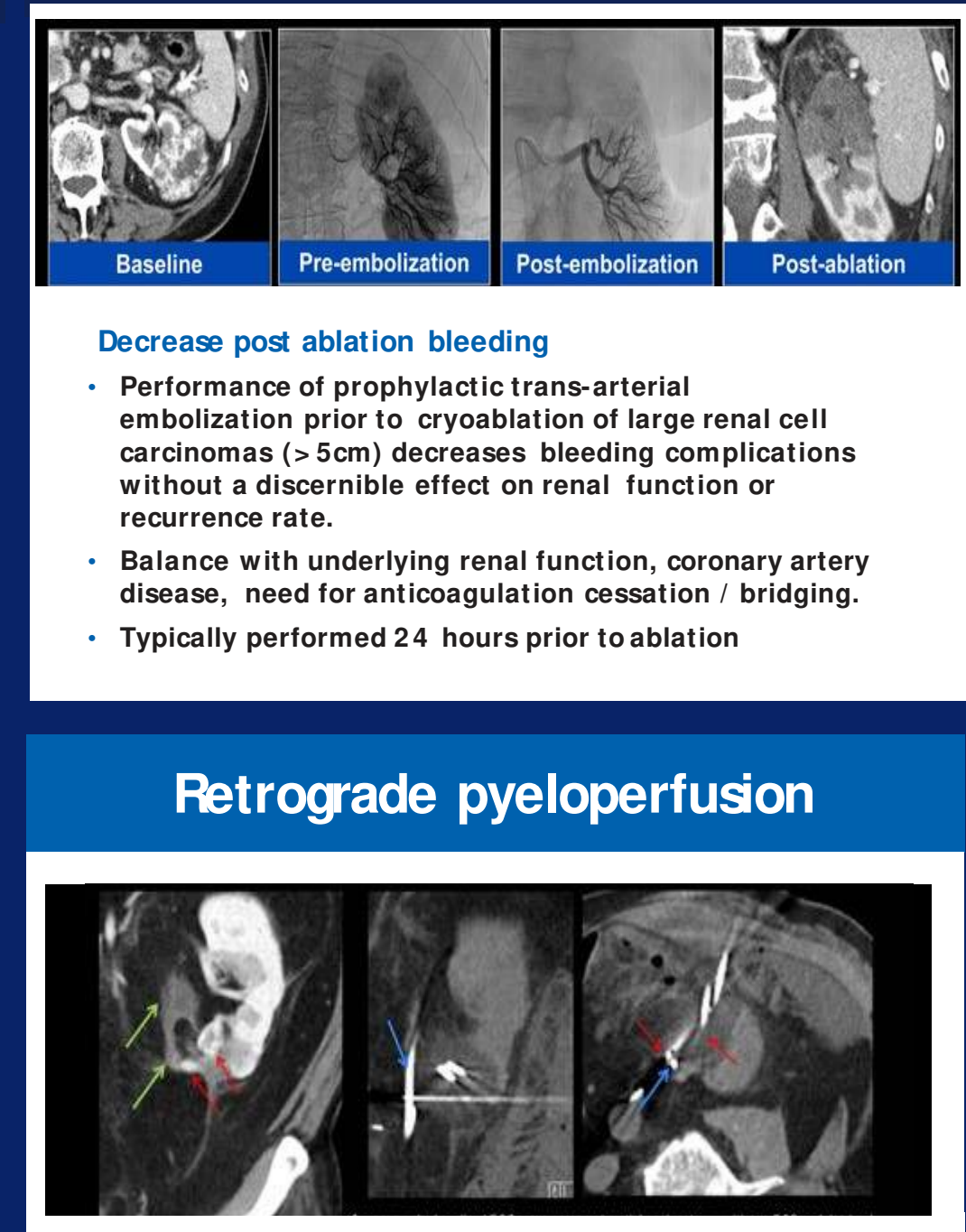

- Ablation performed close to the ureter may result in
ureteral injury and subsequent stricture formation Intraprocedural ureteric / PUJ protection - Intuse
sterile fluid retrogradely via an externalized ureteral stent.
Stent internalization for 6 weeks if ice ball encases the uret Stent internalization for 6 weeks if ice ball encases the
Now $>900$ renal ablations with no ureteral strictures
with this technique.

Urethral warming catheter

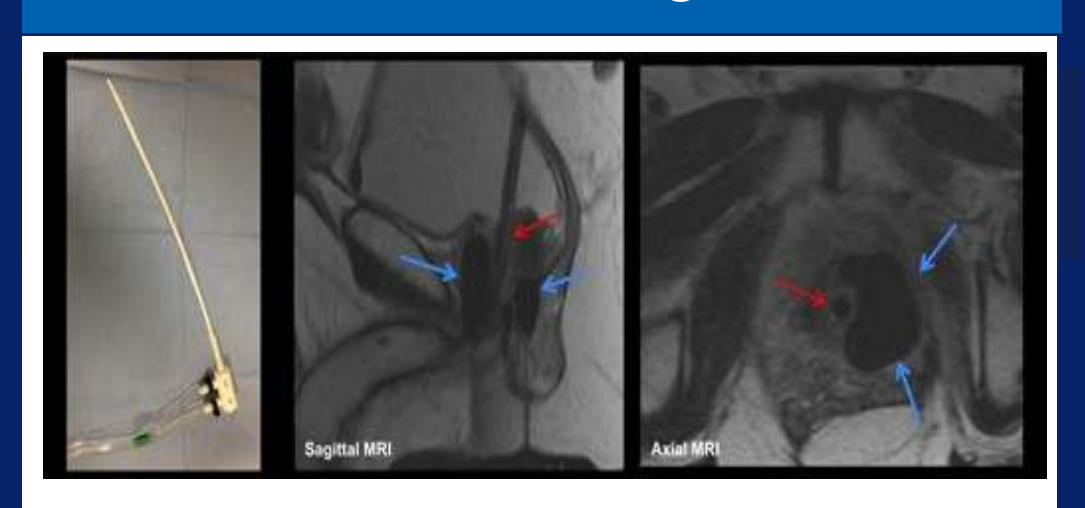

ethral protection

During prostate cryoablation a protective warming
catheter (red arrows i i placed via the urethra into
the bladder prior to commend

Continuous warmed fllid circulates via the closed-loop
continuous- - flow temperature-controlled urethral cathete
during cryoablation to minimize injury of urethral tissue

Significantly decreases risk of se
related to epithelial sloughing.
Adrenal - Adrenergic blockade

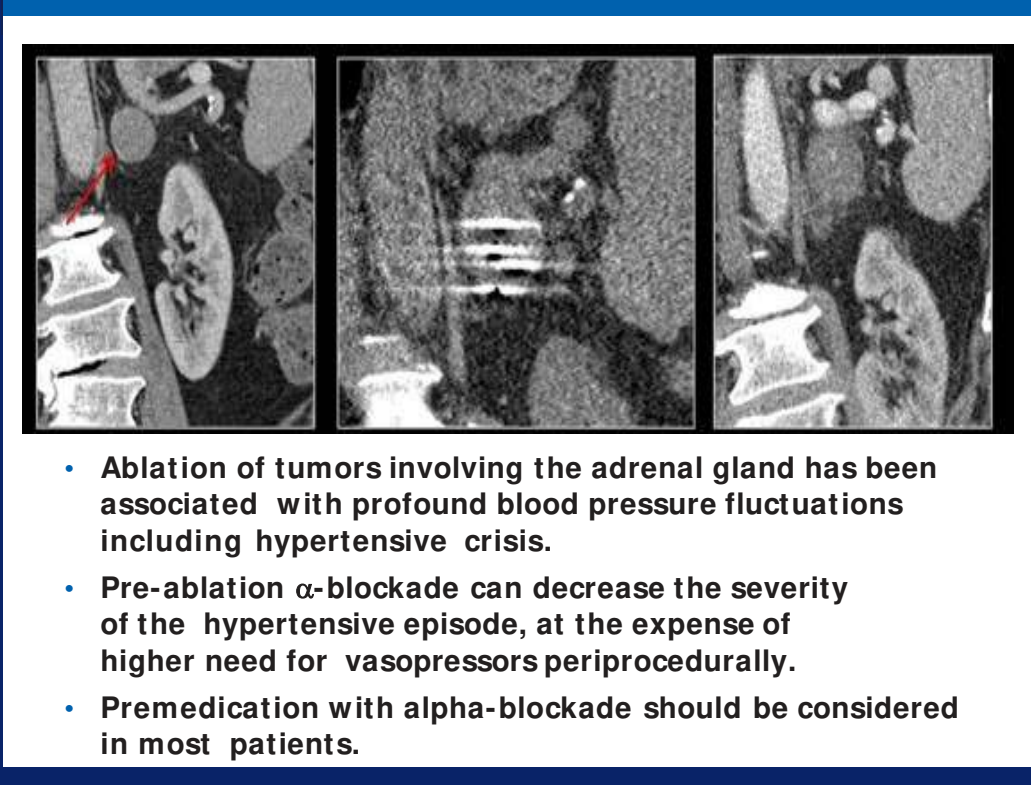

Adrenal - Blood pressure

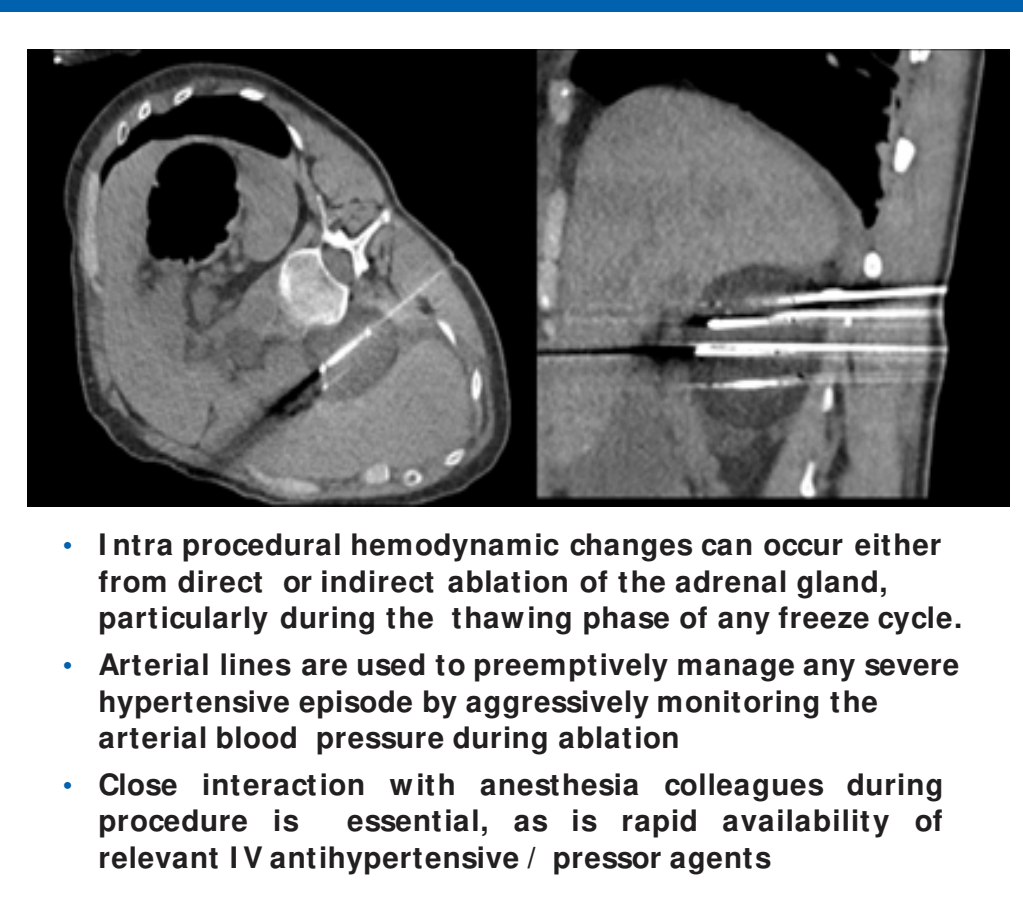

Displacement - Lever Technique

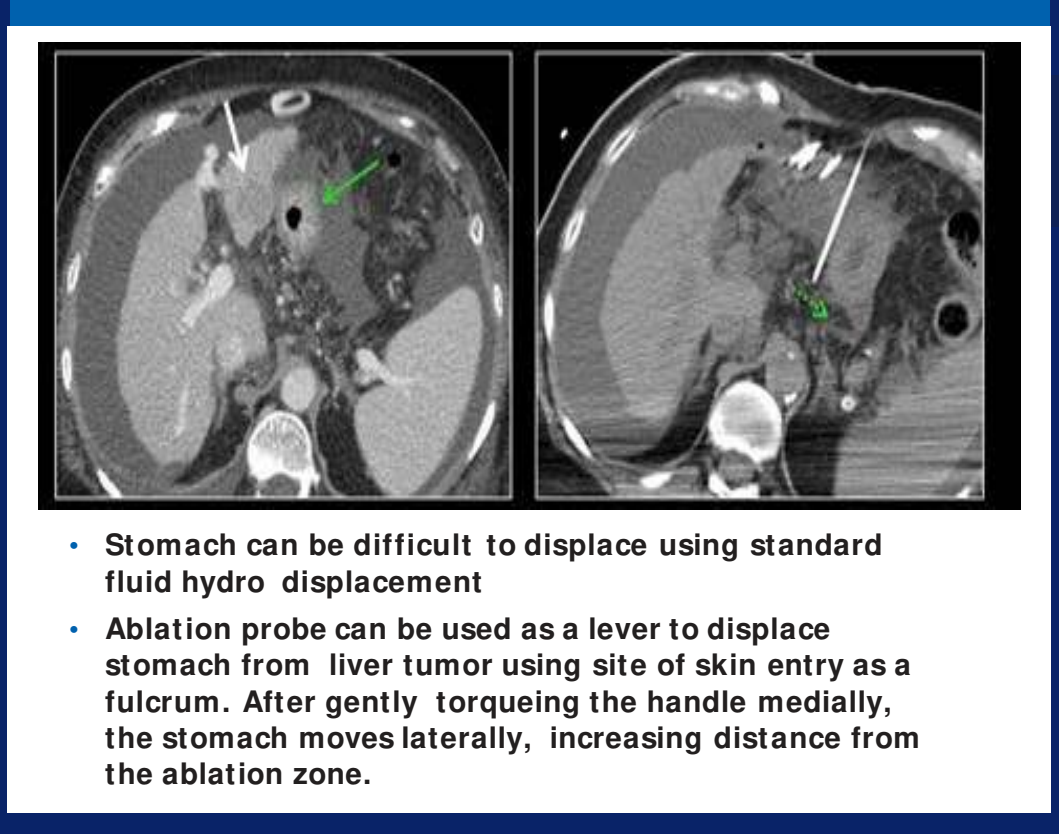

Ultrasound (US) Fusion

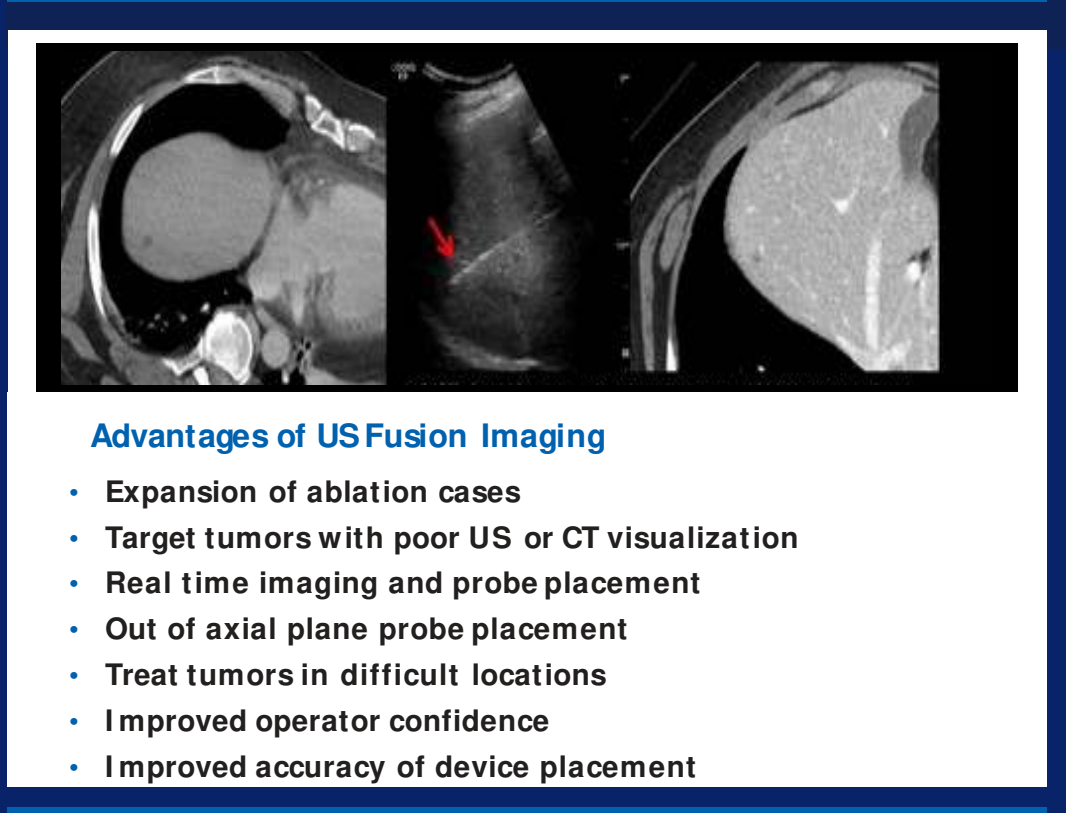

US Fusion Techniques
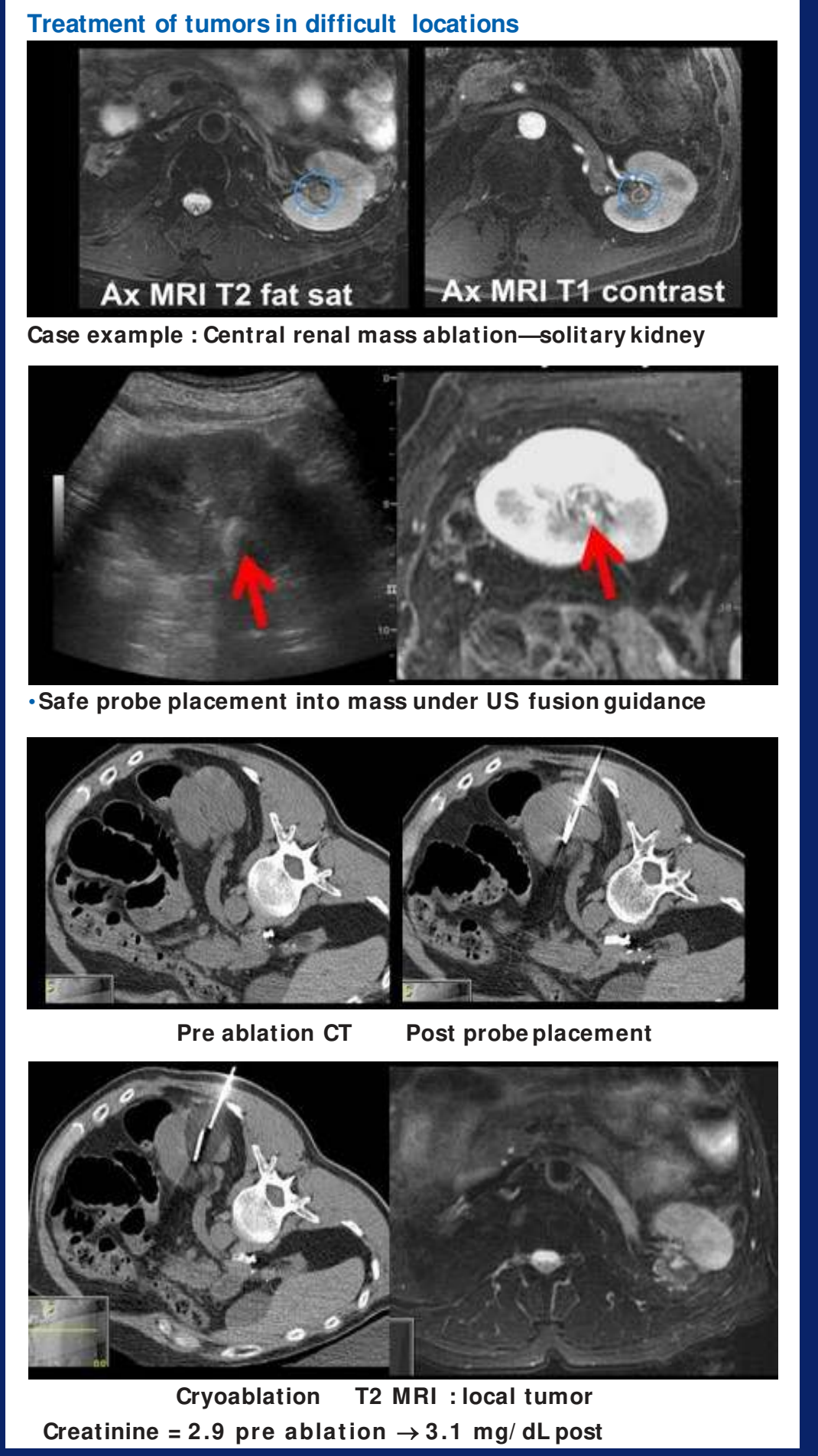

Contrast-enhanced ultrasound

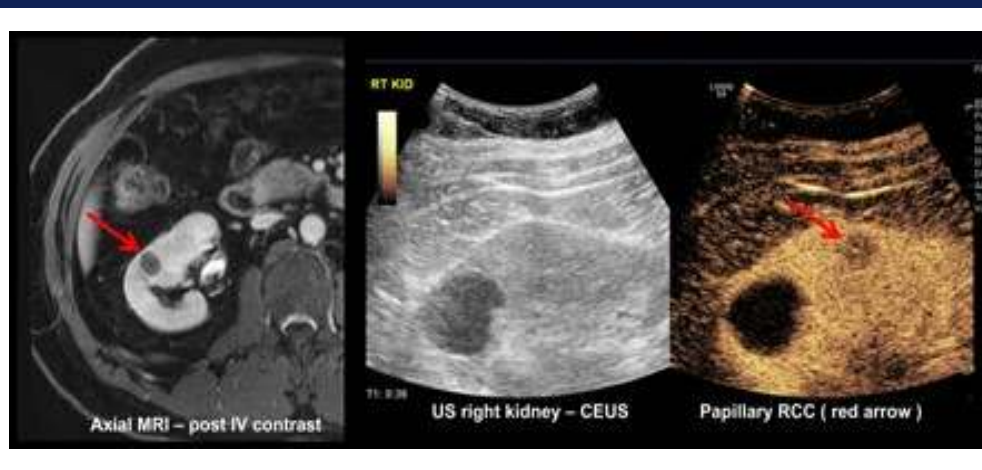

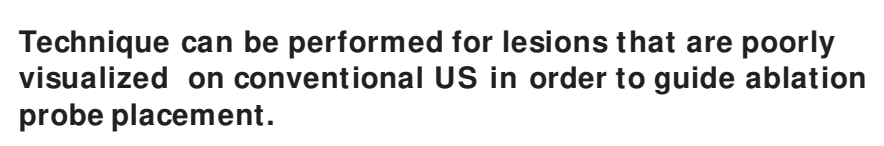
Contrast-enhanced ultrasound (CEUS) involves the
administration of intravenous contrast tagents containing administration of intravenous contrast agents containing
microbubbbles of perfluorocarbon or initrogen gas. The
bubbles affect ultrasound backscatter and increase

CT - Tube current modulation

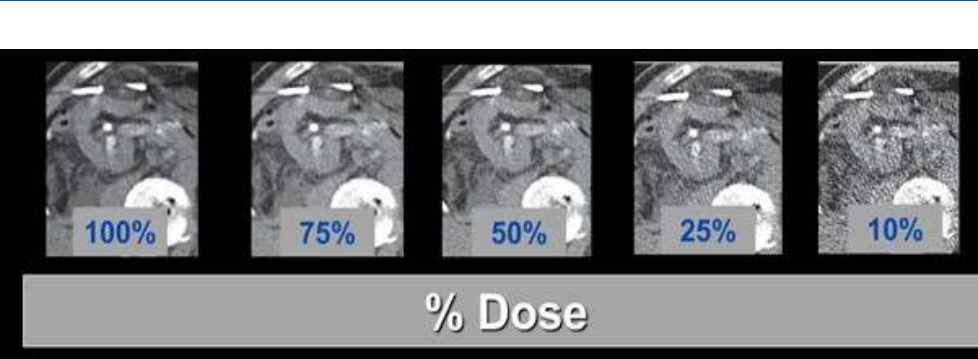

Without compromising the clinical outcome, the smallest
radiation dose should be used to guide and monitor

It has been shown that reduction of mAs by $25-50 \%$
results in inage euality sufficient to effectively
monitor cryoablation.

Summary

After reviewing this exhibit, the participant
should be able to appreciate important
adjunctive techniques related to tumor adjunctiv
ablation

Procedural techniques:

Hydro displacement and other mechanic - ureteral stents to protect collecting system ureteral stents to protect collecting system
Urethral warming catheter to minimize urethra selective embolization to minimize bleeding - alpha blockade for adrenal ablation Imaging techniques:

Imaging techniques: contrast-enhanced ultrasound to identify index

- manual dose reduction during CT monitoring 